\author{
Military Technical College \\ Kobry El-Kobbah, \\ Cairo, Egypt
}

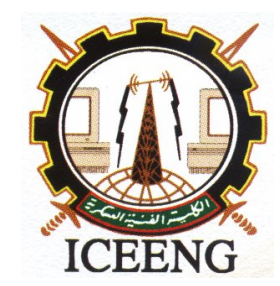

\author{
$7^{\text {th }}$ International Conference \\ on Electrical Engineering \\ ICEENG 2010
}

\title{
Iterative Beamforming Technique for Interference Cancellation
}

$$
\text { By }
$$

Prof. Dr. Khairy El Barbary*

Eng. Basem A. Mansour**

\author{
Dr. Hossam El-Din Abou Bakr* \\ Eng. Haytham Isam E. Yousif ***
}

\begin{abstract}
$\underline{\text { Abstract: }}$
Adaptive beamforming is used in many applications such as radar, sonar and wireless communications for separating desired signals from other unwanted signals. Beamforming is essentially applied to direct the pattern of the receiving antenna system towards the desired direction as well as to attenuate the received signals from unwanted directions. The process of adaptive beamforming is performed in two steps. The first step is assigned to estimate the direction of arrival of signals at the field of view of the receiving system. The second step is assigned to nullify the beam pattern in the directions of unwanted signals. To improve the performance of the adaptive beamforming, the number of array elements as well as the number of snapshots should be increased. However, this increase in both numbers of elements and snapshots leads to increase in the cost and the processing time. In this paper, a proposed iterative adaptive digital beamforming technique is presented to improve the array capability of interference cancellation while reducing the processing time. The proposed technique is based on the Jacobi theorem for iterative solution of a system of linear equation to determine the optimum weight vector at the array output. The proposed technique is compared with the optimal Minimum Variance Distortionless Response (MVDR) beamformer technique. Computer simulation is applied to verify the mathematical analysis that presented in the paper. The paper results show that the proposed technique can provide effective Co-Channel Interference (CCI) suppression better than MVDR, while increasing the strength of the desired signal.
\end{abstract}

\section{Keywords:}


Adaptive beamforming, Minimum Variance Distortionless Response (MVDR), CoChannel Interference (CCI)

$\begin{aligned} * & \text { Egyptian Armed Forces } \\ * * & \text { Syrian Armed Forces } \\ * * * & \text { Sudanese Armed Forces }\end{aligned}$

\section{Introduction:}

A major challenge for wireless communications systems is the limited capacity due to sparse radio frequency spectrum. The capacity limitation, in practice, is mainly determined by the capability of interference cancellation. One of the serious types of interference is the Co-Channel Interference (CCI) when the unwanted signals have the same carrier frequency of the desired signal. The matched filter (in temporal domain) is failed to solve this problem. As a remedy, the beamforming is proposed as spatial filter for the purpose of interference cancellation. Beamforming is a general signal processing technique used to control the directionality of the reception or transmission of a signal on a transducer array. The beamforming adjust the weighting vector of the array element to determine the optimum weight vector.

In the fixed weight beamforming approach the arrival angles do not change with time, so the optimum weight would not need to be adjusted. However, if desired arrival angles change with time, it is necessary to devise an optimization scheme that operates on the automatic changing so as to keep recalculating the optimum array weight, that's done by using adaptive beamforming algorithm [1],[2].

Interference cancellation is a simple multi-user detection technique, in which the estimates of other users interference are subtracted from the received signal, in order to improve the desired user estimate [3], [7]. This process can be performed iteratively by repeating the subtraction process, using estimates from previous stages to improve the estimates at subsequent stages. Iterative techniques, such as interference cancellation may be linear or non linear. Linear techniques use linear estimates of the interference, whereas non-linear techniques have no such restriction.

Due to the system complexity, the fabrication cost and the operational expenditure, adaptive antennas are limited to base stations or military applications. Researchers are endeavoring to make low profile and low power consumption antennas for mobile terminals [11]. Some new approaches in adaptive beamforming techniques are proposed in [12-17].

In this paper, the linear techniques is only considered, a new low complexity iterative technique is proposed to steering null to the Co-Channel Interference (CCI) locations while forming beam to the desired direction without the use of temporal 
reference signal. In this paper iterative techniques are used in spatial reference beamforming area.

This paper provides analytic evaluation of the proposed technique for interference cancellation as well as computer simulation for its behavior for different situations. Sections 2 and 3 provide brief description as well as analytic derivation of optimal Minimum Variance Distortionless Response (MVDR) beamformer technique and Iterative Null Steering technique for interference cancellation, respectively. Section 4 provides computer simulation results for the behavior of the two techniques and comparison between them. Finally section 5 provides conclusions and recommendations for future work.

\section{Optimal Beamforming}

Consider $K$ received narrowband signals from different directions $\theta_{1}, \ldots, \theta_{K}$ impinging a uniform linear array (ULA) composed of $M$ sensors. The outputs observation from the array elements, separated by the sampling interval of the process are denoted by $X(1), X(2), \ldots, X(L)$ each observation is a complex vector of $M$ elements and it is called snapshot. The total number of available snapshots is assumed to be $L$ snapshots. The $M \times L$ array observation matrix is modeled as

$$
X(t)=\mathbf{A}(\theta) \mathbf{S}(t)+\mathbf{n}(t)
$$

where, $\mathbf{A}(\theta)=\left[a\left(\theta_{1}\right), \cdots, a\left(\theta_{K}\right)\right]$ is the array response matrix, $\mathbf{s}(\mathrm{t})$ is the transmitted signals vector at time instant $\mathrm{t}$ and $\mathbf{n}(t)$ is the noise vector. The $m^{\text {th }}$ column of the steering matrix is defined as:

$$
\mathbf{a}\left(\theta_{m}\right)=\left[1, \exp \left(-j \phi_{m}\right), \exp \left(-j 2 \phi_{m}\right), \ldots, \exp \left(-j(M-1) \phi_{m}\right)\right]^{T} ; 1 \leq m \leq K
$$

where $[.]^{T}$ denotes transpose and the inter-element phase shift is defined as $\phi_{m}=\left(\frac{2 \pi d}{\lambda}\right) \sin \theta_{m}$, where $d$ is the inter-element spacing of the array, $\lambda$ is the operating wavelength.

The spatial correlation matrix of the observed signal vector is defined as:

$$
R_{x x}=E\left[x(t) x(t)^{H}\right]
$$

where $\mathrm{E}[$.$] is the expectation matrix, [.] { }^{H}$ is Hermitian (complex conjugate transpose). Then substitute equation (1) into (3) and due to the signal and noise are uncorrelated:

$$
R_{x x}=A(\theta) E\left[s(t) s(t)^{H}\right] A^{H}(\theta)+E\left[n(t) n(t)^{H}\right]
$$


Assume that the noise vector is additive white Gaussian noise (AWGN) with zero mean and a variance of $\sigma^{2}$, then equation (4) becomes

$$
R_{x x}=A(\theta) R_{S} A^{H}(\theta)+\sigma^{2} \mathrm{I}_{(M)} .
$$

where $R_{s}$ is the correlation matrix of the transmitted signals and $\mathrm{I}_{(\mathrm{M})}$ is identity matrix of order $\mathrm{M}$.

The array output is a linear weighted combination of the sensor outputs

$$
Y_{B}(t)=W^{H} X(t)
$$

where $\mathrm{W}$ is the weight vector of the array outputs as shown in figure.1.

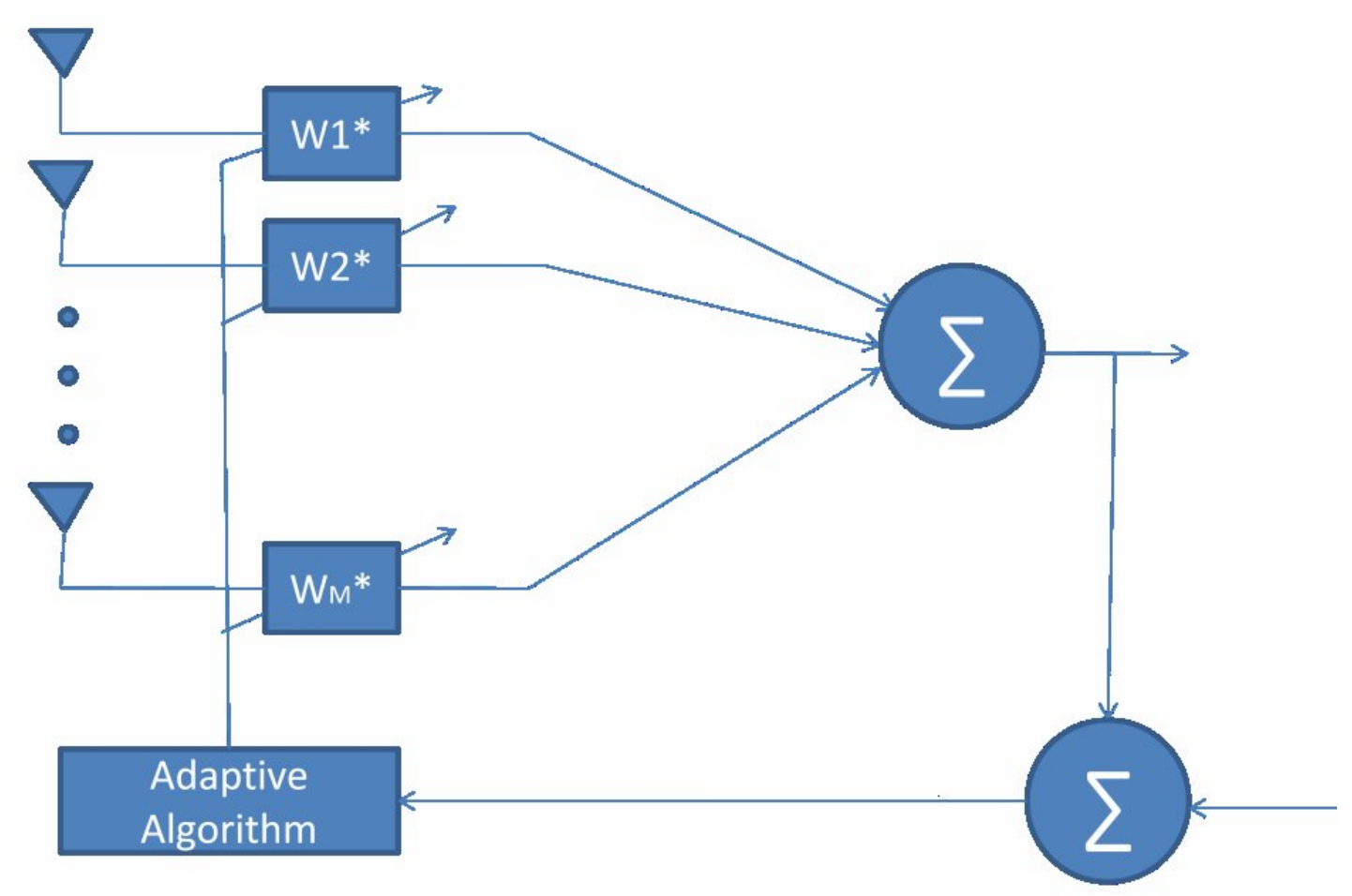

Figure 1. Block diagram of adaptive beamforming system

Suppose the desired signal is arriving from a direction $\theta_{1}$, then the optimal beam forming weight vector, $w_{B F}$, is derived as follows:

The array output vector for a single source coming from direction $\theta_{1}$ can be written as:

$$
x^{(1)}(t)=\mathbf{a}\left(\theta_{1}\right) S_{1}(t)+\mathbf{n}(t)
$$


Since the noise vector is (AWGN) and it is independent of the input signal vector, then the array output power is given by:

$$
\begin{aligned}
P_{B F}\left(\theta_{1}\right) & =E\left\{\left|Y_{B}(t)\right|^{2}\right\}=E\left[w^{H} x^{(1)}(t) x^{(1)}(t)^{T} w\right] \\
& =w^{H} R_{x x}^{(1)} w=w^{H}\left[a\left(\theta_{1}\right) R_{s_{1}} a^{H}\left(\theta_{1}\right)+\sigma^{2} I\right] w \\
& =w^{H} a\left(\theta_{1}\right) R_{s_{1}} a^{H}\left(\theta_{1}\right) w+w^{H} \sigma^{2} w
\end{aligned}
$$

In case of multiple sources, the array output power is given by:

$$
P_{B F}(\theta)=w^{H} a\left(\theta_{1}\right) R_{s_{1}} a^{H}\left(\theta_{1}\right) w+\sum_{i=2}^{k} w^{H} a\left(\theta_{i}\right) R_{s_{i}} a^{H}\left(\theta_{i}\right) w+w^{H} \sigma^{2} w
$$

Since it is desired to steer nulls in the directions of sources $s_{i} ; i=2,3, \ldots \mathrm{k}$

while maintaining the output power due to the desired source as max, then this problem is equivalent to the constrained problem

Condition that

$$
\min _{w}\left(w^{H} R_{x x} w\right)
$$

$$
\left|w^{H} a\left(\theta_{1}\right)\right|=1
$$

For a positive definite correlation matrix, the solution of equation (10) of the weight vector can be readily given by [4], [5].

$$
w_{M V D R}=\frac{R_{x x}^{-1} a\left(\theta_{1}\right)}{a^{H}\left(\theta_{1}\right) R_{x x}^{-1} a\left(\theta_{1}\right)}
$$

The weight obtained by equation (11) is called the Minimum Variance Distortionless Response (MVDR). Using the weight vector $w_{M V D R}$, the array output power spatial spectrum has the form:

$$
P_{C}(\theta)=\frac{1}{a^{H}\left(\theta_{1}\right) R_{x x}^{-1} a\left(\theta_{1}\right)}
$$

\section{Iterative Null Steering}

In many applications the reaction for the information gathered by the array should be in real time and there isn't enough number of snapshots. Thus, a proposed technique is introduced to deal with minimum number of snapshots of the received signals and without the use of temporal reference signal to adjust the weight vector to the desired response. The proposed technique should have a lower processing time compared to the 
MVDR beam former.

For enhancement of the desired signal, arriving from direction $\theta_{1}$, and nullifying the other unwanted directions, the following system of linear equations, which is equivalent to the constrained optimization problem in equation (10), should be solved:

$$
R_{x x} w^{H}=a\left(\theta_{1}\right)
$$

The calculations of unknown vector $w^{H}$ requires in general $O\left(n^{3}\right)$ operations (unless $R_{x x}$ has some special property that makes it easily invertible). Equation (13) can be solved iteratively based on the Jacobi theorem [7] which derived in next subsection.

\subsection{Jacobi Theorem}

The system of linear equations is defined as

$$
\mathbf{H x}=\mathbf{b}
$$

Could be solved using iteration technique, that

$$
x_{k+1}=G x_{k}+c
$$

where

$$
G=-D^{-1}[L+U]
$$

and

$\mathrm{C}=D^{-1} b$

The matrices $\boldsymbol{D}, \boldsymbol{L}$ and $\boldsymbol{U}$ are the diagonal, strictly lower triangular and strictly upper triangular parts of $\mathbf{H}$ respectively. A sufficient condition for the method to be applicable is that $\mathbf{H}$ is strictly diagonally dominant or diagonally dominant and irreducible.

The sufficient conditions for Jacobi iteration to provide convergence are:

1-The initial value $x_{o}$ must be selected carefully to guarantee the sequence $\left\{x_{k}\right\}$ converge to the suitable solution.

2- The spectral radius ( $\rho$ ) of the matrix $G$ in equation (15) must be less than 1 . This is equivalent to say that, the magnitude of the largest eigenvalue $\lambda_{\max }$ of $\mathbf{H}$ must be less than one.

The proof of the Jacobi theorem and convergence of the iteration technique could be explained as follows:

The solution of the system of equation (14) is given by

$$
\mathbf{x}=\mathbf{H}^{-1} \mathbf{b}
$$

where $\mathrm{H}^{-1}$ is the inverse matrix of $\mathbf{H}$, which satisfy the relation

$$
\mathbf{H}^{-1}=\mathbf{I} / \mathbf{H} \stackrel{\Delta}{=} \mathbf{I} / \mathbf{I}-\mathbf{E}
$$

where $\mathbf{E}$ is the processing error matrix. If the norm of the matrix $\mathbf{E}$ is less than one, then by applying Taylor series expansion of (17) we can write that

$$
\mathbf{H}^{-1}=\mathbf{I}+\mathbf{E}+\mathbf{E}^{\mathbf{2}}+\mathbf{E}^{3}+
$$

Since the norm of $\mathbf{E}$ is less than one we can neglect the higher order terms of (18) 


$$
\mathbf{H}^{-1} \approx \mathbf{I}+\mathbf{E}
$$

Consequently equation (15) is proved

\subsection{Application of Jacobi Theorem on the Adaptive Beamforming}

In our case the iterative solution of equation (13) is

$$
w_{k+1}=-D^{-1}(L+U) w_{k}+D^{-1} a\left(\theta_{1}\right)
$$

From the matrix algebra

$$
(L+U)=\left(R_{x x}-D\right)
$$

Assume that $D \approx I$, then from equations (19) and (21), the equation (20) becomes

$$
\begin{aligned}
w_{k+1} & =-\left(R_{x x}-I\right) w_{k}+a\left(\theta_{1}\right) \\
& =a\left(\theta_{1}\right)-\left(R_{x x}-I\right) w_{k}
\end{aligned}
$$

The initial guess of $w_{0}=a\left(\theta_{1}\right)$ leads to

$$
w_{k}=\sum_{i=0}^{k}(-1)^{i}\left(R_{x x}-I\right)^{i} a\left(\theta_{1}\right)
$$

The convergence of this iteration in equation (20) may be understood by considering the residual error in terms of the Taylor series expansion [7]

$$
R_{x x}^{-1}=\sum_{i=0}^{\infty}(-1)^{i}\left(R_{x x}-I\right)^{i}, \quad \rho\left(R_{x x}\right)<2
$$

The convergence occurs if the spectral radius of $R_{x x}$ is lower than 2 .

A simple generalization which improves on the Jacobi-style iteration is brought about by the introduction of a parameter or a sequence of parameters. By carefully selecting these parameters, the convergence speed can be improved.

A first order iteration is given by [7]:

$$
w_{k+1}=w_{k}-\lambda_{k}\left(R_{x x} w_{k}-a\left(\theta_{1}\right)\right)
$$

where $\lambda_{k}$ is step of convergence for iteration technique.

As $R_{\mathrm{xx}}$ is symmetric positive definite, then the parameter that results in fastest convergence of the first order stationary iteration is

$$
\lambda_{\text {opt }}=\frac{2}{\lambda_{\min }+\lambda_{\max }}
$$

where $\lambda_{\min }$ and $\lambda_{\max }$ are respectively the minimum and the maximum eigenvalues of $R_{x x}$. A second order iteration that depends on last two estimates is given by:

$$
w_{k+1}=\alpha_{k} w_{k}+\left(1-\alpha_{k}\right) w_{k-1}-\beta_{k}\left(R_{x x} w_{k}-a\left(\theta_{1}\right)\right)
$$

Where the best choice of $\alpha$ and $\beta$ are given in [7]: 


$$
\begin{aligned}
& \alpha_{o p t}=\frac{2}{1+\sqrt{1-\left(\frac{1-\left(\lambda_{\min } / \lambda_{\max }\right)}{1+\left(\lambda_{\min } / \lambda_{\max }\right)}\right)^{2}}}, \\
& \beta_{\text {opt }}=\frac{2 \alpha_{o p t}}{\lambda_{\min }+\lambda_{\max }} .
\end{aligned}
$$

The Chebychev technique is another iterative technique in which the optimal $\lambda_{k}$ is known for a given number of iteration steps. The optimum value for $\lambda_{i}$ is given by [8]:

$$
\lambda_{k_{\text {opt }}}=\frac{\lambda_{\max }-\lambda_{\min }}{2} \cos \left(\frac{k-1 / 2}{k_{\max }+1} \pi\right)+\frac{\lambda_{\max }+\lambda_{\min }}{2}
$$

\section{Simulation Results}

Computer simulations are performed using MATLAB to verify the theoretical background obtained in the pervious sections. In the simulations two interference signals are located at $-20^{\circ}, 60^{\circ}$ from the array bore sight and one desired signal is located at $20^{\circ}$ from the array bore sight present in the instantaneous view of the uniform linear antenna array. The antenna array consists of 4 elements and the spacing between them is considered to be one fourth the wave length corresponds to the used carrier frequency. The signals are considered to be narrowband signals each signal possess a $\mathrm{SNR}=10 \mathrm{~dB}$.

Figures 2, 3 compare between performances of first order iterative technique after 10, 50 snapshots respectively and the optimum MVDR beam former after 256 snapshots. One can see that the first order iteration provides deeper null than the MVDR beam former in second case, moreover as the number of available snapshots increase the deep of the nulls increase.

Similarly figures 4, 5 compare between performances of second order iterative technique for 10 snapshots and 50 snapshots, respectively, and the optimum MVDR beam former after 256 snapshots. Clearly the second order iteration achieves deeper null compared to MVDR and first order iteration. That is due to fast convergence of second order compared to first order iteration.

Finally figure 6 compare between performances of Chebychev iterative technique and the optimum MVDR beam former, results show that the Chebychev iterative technique achieves deeper null compared to MVDR, first order iteration and second order iteration, respectively.

In terms of the peak at 20 degrees and null at -20 and 60 degrees, the conventional optimum beam former method achieves the best nulls but the worst peak. The Chebychev method performs best, both in terms of Null and peak and in terms of providing a fast convergence. 


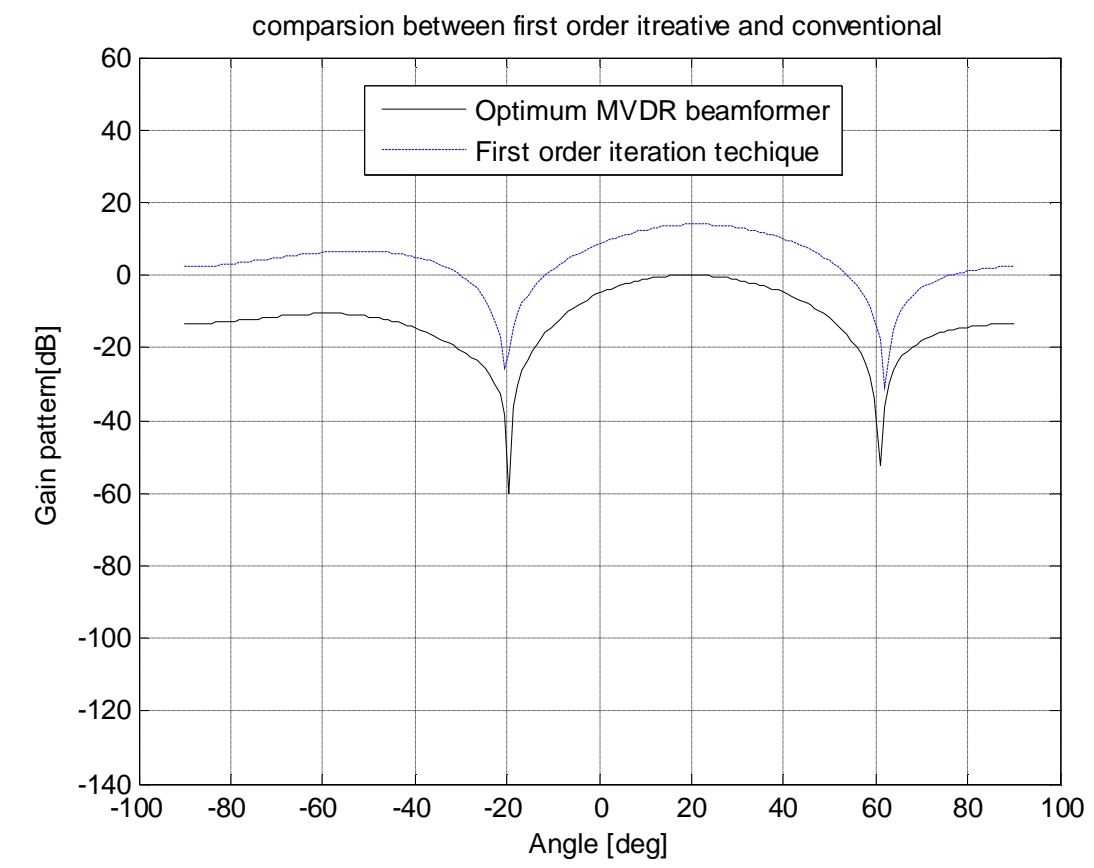

Figure (2): Comparison between the optimum MVDR beamformer after 256 snapshots and first order iterative technique after 10 iterations.

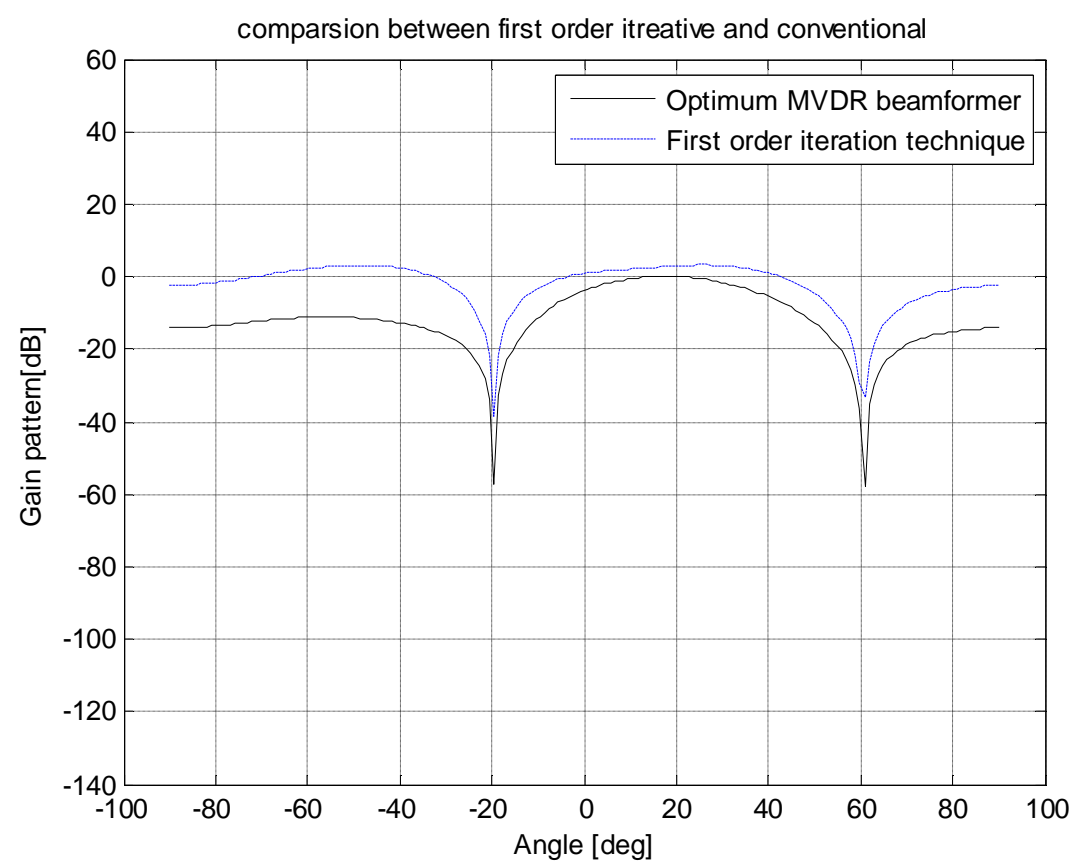

Figure (3): Comparison between the optimum MVDR beamformer after 256 snapshots and first order iterative technique after 50 iterations. 


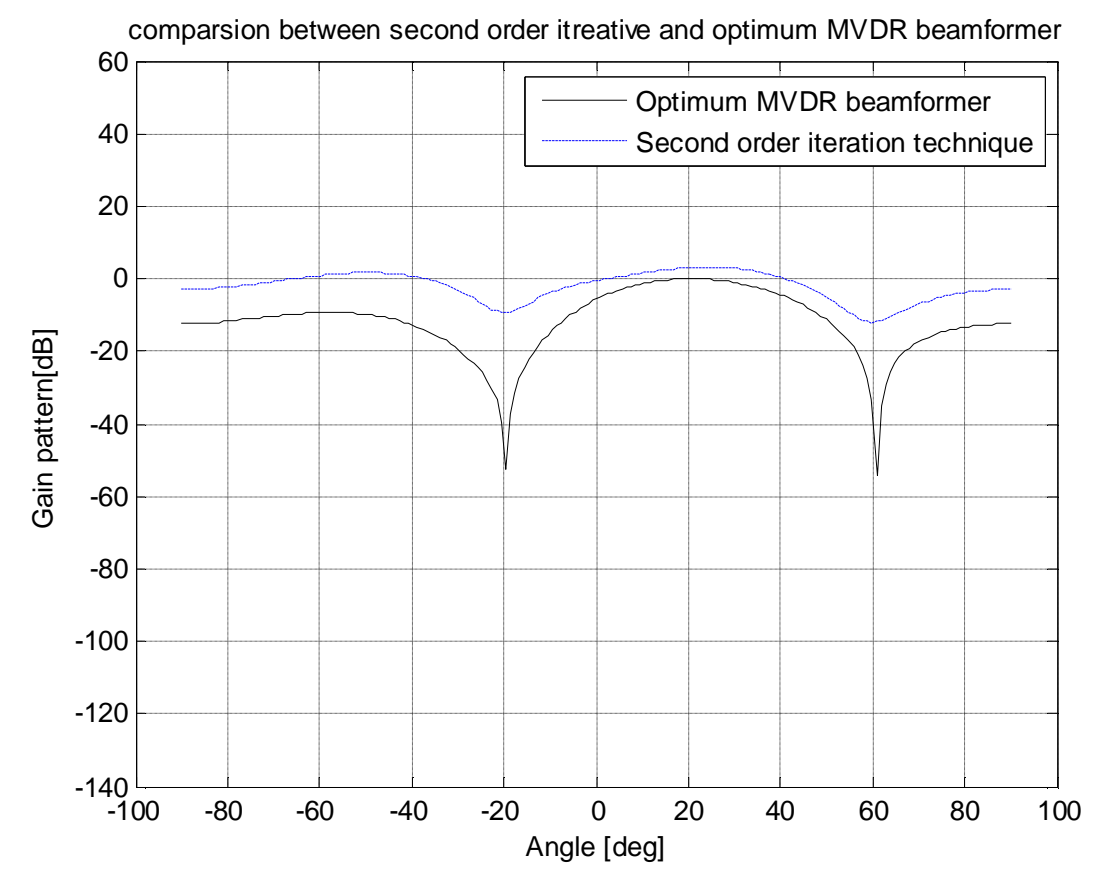

Figure (4): Comparison between the optimum MVDR beamformer after 256 snapshots and second order iterative technique after10 iterations.

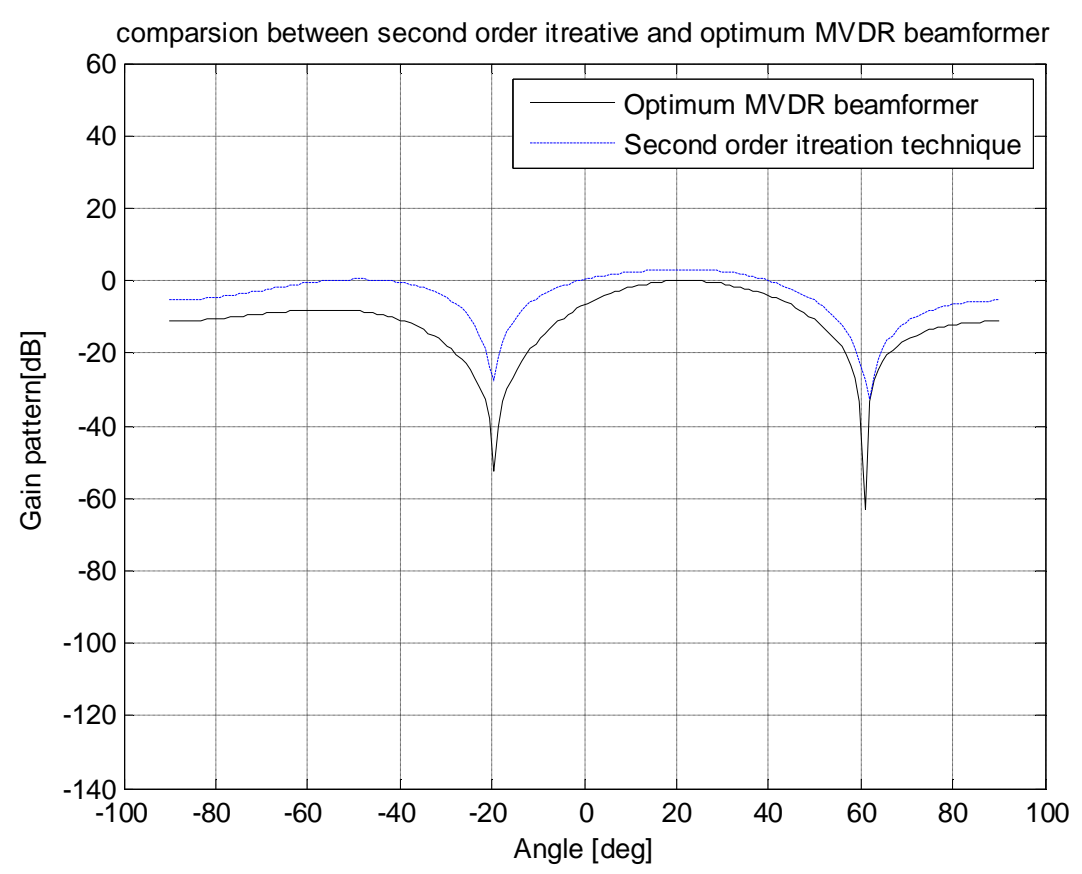

Figure (5): Comparison between the optimum MVDR beamformer after 256 snapshots and second order iterative technique after 50 iterations. 


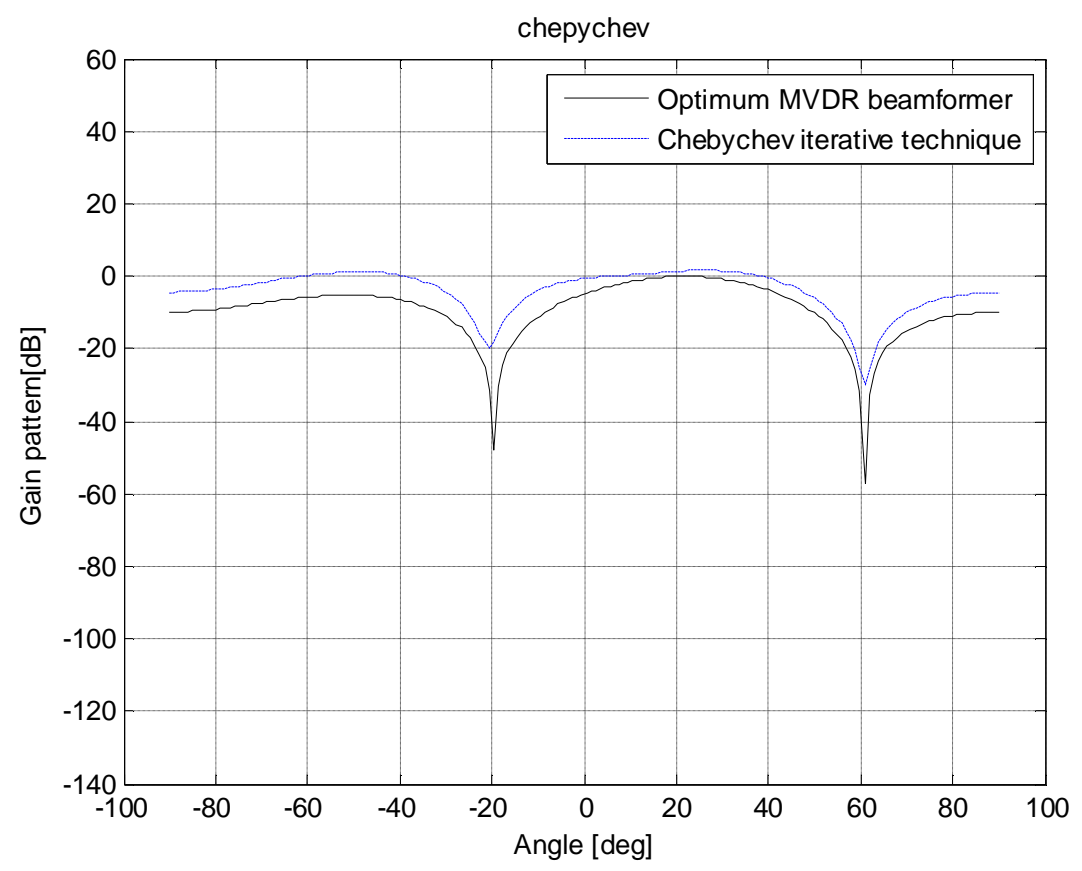

Figure (6): Comparison between the optimum MVDR beamformer after 256 snapshots and first order Chebychev iterative technique after10 iterations.

\section{Conclusions}

This paper presented a low complexity and computationally efficient iterative beamforming technique for interference cancellation. This technique is independent of the number of sources as shown in its mathematical model. The proposed iterative techniques are used to solve the limits of the conventional inverse matrix calculation. The simulation results show that this technique converges faster than the conventional MVDR beamformer with minimum number of snapshots. The iterative method dose not require reference signal. Rather it works on the real received data. We want to extent this work to determine the accuracy and resolution of the proposed beamformer. Also the effect of motion of the emitting source on the perform will be considered. 


\section{References}

[1] Singh, A. K., P. Kumar, T. Chakravarty, G. Singh, and S. Bhooshan, "A novel digital beamformer with low angle resolution for vehicle tracking radar," Progress In Electromagnetics Research, PIER 66, 229-237, 2006.

[2] Barry D., Van Veen and Kevin M. Buckley, "Beamforming: A Versatile Approach to Spatial Filtering”, IEEE ASSP Magazine, pp.4-24, April 1988.

[3] Hamid Krim, Mats Viberg, "Two Decades of Array Signal Processing Research ", IEEE Signal Processing Magazine, pp.67-94, July 1996.

[4] Applebaum, S. P., "Adaptive arrays," IEEE Trans. Antennas Propagat., Vol. 24, 585-598, 1976.

[5] Bresler, Y., V. U. Reddy, and T. Kailath, "Optimum beamforming for coherent signal and interferences," IEEE Trans. Acoust. Speech Signal Process., Vol. 36, 833-843, 1988.

[6] Gilbert Strang, Introduction to Linear Algebra, third adition, WellesleyCambridge Press, 2003.

[7] Grant, A. and C. Schlegel, "Iterative implementation for linear multiuser detectors," Department of Electrical Engineering, University of Utah, 1999.

[8] M. Emadi, K. H. Sadeghi, A. Jafargholi and F. Marvasti, "Co-Chanel Interfernce cancellation by the use of Iterative digital beamformer method," Progress In Electromagnetics Research, PIER 87, 89-103, 2008.

[9] Marvasti, F., "An iterative method to compensate for the interpolation distortion," IEEE Trans. ASSP, Vol. 3, No. 1, 1617-1621, 1989.

[10] Marvasti, F., M. Analoui, and M. Gamshadzahi, "Recovery of signals from non uniform samples using iterative methods," IEEE Trans. ASSP, Vol. 39, No. 4, 872-878, Apr. 1991.

[11] Mouhamadou, M. and P. Vaudon, "Smart antenna array patterns synthesis: Null steering and multi-user beam forming by phase control," Progress In Electromagnetics Research, PIER 60, 95-106, 2006.

[12] Pedersen, K. I., P. E. Mogensen, and B. H. Fleury, "A stochastic model of the temporal and azimuthal dispersion seen at the base station in outdoor propagation environments," IEEE Trans. Veh. Technol., Vol. 49, No. 2, 437-447, 2000.

[13] Vorobyov, S. A., A. B. Gershman, and Z. Q. Luo, "Robustadaptive beamforming using worst-case performance optimization:A solution to the signal mismatch problem," IEEE Trans. Signal Processing, Vol. 51, No. 2, 313-324, 2003.

[14] Li, J., P. Stoica, and Z. Wang, "On robust capon beam forming and diagonal loading," IEEE Trans. Signal Processing, Vol. 51, No. 7, 1702-1715, 2003. 
[15] Lorenz, R. G. and S. P. Boyd, "Robust minimum variance beam forming," IEEE Trans. Signal Processing, Vol. 53, No. 5, 1684-1696, 2005.

[16] Besson, O., A. A. Monakov, and C. Chalus, "Signal waveform estimation in the presence of uncertainties about the steering vector," IEEE Trans. Signal Processing, Vol. 52, No. 9, 2432-2440, 2004. 
